\title{
Acquisition of an Electronic Patient Record System
}

\author{
By Daniel B. Gordon and Samuel Marafioti
}

S unnybrook Health Science Centre in Toronto recently conducted an extensive cost-benefit analysis to evaluate the merits of implementing an Electronic Patient Record System. The following describes some of the calculations which were essential to the decision-making process.

As a first step, the organization underwent a rigorous strategic planning process. The resulting plan identified a number of strategic reasons why Sunnybrook Health Science Centre should embark on an electronic patient record implementation process. A portion of the plan identified the stakeholders of the Electronic Patient Record and the benefits of the proposed system to each stakeholder group.

This strategic plan suggested that among the benefits of the project would be improved quality of care and better risk management. Unfortunately, it was not easy to quantify specific benefits with dollar values. The costs, by contrast, were much easier to enumerate and calculate. For instance, the plan recommended a phased-in approach to the overall implementation (to last approximately five years) with a multi-million-dollar requirement applied to the project in its first year. It was generally outlined that economic benefits might be derived from more efficient patient and process management, but financial returns were not anticipated during the first five years. The Electronic Patient Record strategic plan was approved at the most senior levels of the organization.

As a background, two additional systems-related challenges faced the hospital at this time. First, the year 2000 was rapidly approaching, necessitating the remediation or replacement of all non-Year 2000 compliant systems, including the mainframe patient care system. Second, Ontario hospital restructuring had begun and Sunnybrook Health Science Centre was made aware that there would be significant-systems integration challenges as a result of any proposed changes. Finally, the Electronic Patient Record strategic plan was designed to replace the main hospital clinical care systems, which were not Year 2000 compliant .

\section{Vendor Selection Process}

Following the approval of the Electronic Patient Record strategic plan Sunnybrook Health Science Centre embarked on a vendor selection process. The focus of this process was on finding a commercially available system that multiple stakeholders agreed would meet their needs and that fitted with Sunnybrook Health Science Centre's strategic direction. At the end of this process, a vendor was selected and both Year 2000 and restructuring issues were dealt with in subsequent contractual negotiations.

The next step was to obtain organizational and Board-level approval of the budget required for this project. A five-year budget was developed. Cost line items included hardware, hardware support, clinical workstations, software licences, software support and implementation services, project management, dedicated project staff, staff training, and backfill funding to replace staff during training. Actual savings were identified in the area of reduced paper filing and transcription, and the elimination of maintaining an out-sourced mainframe data centre. In the strategic plan these savings were not projected to begin until year five at the earliest. The five-year Electronic Patient Record budget was amalgamated into an overall Information Systems budget, which included line items for other Year 2000 replacement systems and network infrastructure to support this new environment. This was the budget presented for approval.

\section{Analysis of Care Efficiencies}

As supporting evidence, Sunnybrook also did an analysis of the care efficiencies that might accrue from this project. Savings resulting from changes in the way physicians write orders included a reduced number of tests thanks to test history feedback; test ordering menus that favour selective problem-oriented testing and longer intervals between tests; a reduced pharmacy budget thanks to drug-cost feedback; drug use guidelines; and shorter lengths of stay thanks to reduced delays in test scheduling, electronic drug-order delivery, and more efficient clinical decision making. Potential dollar savings were projected based on reported clinical-trial evidence (Tiernay et al. 1993). As hard figures for these benefits were not available, the analysis included many estimates. The main factor in demonstrating that the benefits outweighed the costs was the inclusion of the estimated cost of remediating Sunnybrook's current mainframe system to become Year 2000 compliant. This effort would still be required if the system replacement strategy were not approved. As a result, these two savings and cost-avoidance figures were presented to the Board in support of the Electronic Patient Record initiative. The strategic plan was approved.

In conclusion, Sunnybrook Health Science Centre's costbenefit analysis for acquiring its Electronic Patient Record system used a combination of strategic need, actual dollar costs, actual savings, projected savings and projected cost avoidance. The analysis demonstrated that the project would not be cost-neutral in its first years. The Board agreed that the investment was necessary for strategic reasons: to allow Sunnybrook to maintain its reputation for leading-edge information systems and to position the organization for the year 2000 and for merger challenges. IQP

Daniel B. Gordon, Ph.D., Sunnybrook Health Sciences Centre Samuel Marafioti, M.Sc., Sunnybrook Health Science Centre 